\title{
Multimorbidität und chronische Krankheiten prägen zusehends den ärztlichen Alltag
}

\author{
Multimorbidität und chronische Krankheiten fordern von Ärzten, insbesondere von \\ Generalisten, komplexe diagnostische und therapeutische Überlegungen, die es zu \\ erforschen gilt. Der diagnostische und therapeutische Zugang wird sich aufgrund \\ dieser Forschung verändern, und die Betreuung multimorbider Patienten wird \\ Hauptthema der Gesundheitsversorgung der Zukunft sein.
}

\section{Edouard Battegay}

Direktor Klinik und Poliklinik für Innere Medizin, UniversitätsSpital Zürich, Tagungspräsident der 81. SGIM-Jahresversammlung 2013

Die französische Fassung dieses Artikels erschien am 20. Februar 2013 in der Revue Médicale Suisse:

Battegay E. La multimorbidité et les maladies chroniques façonnent toujours plus le quotidien des médecins. Rev Méd Suisse. 2013;9:430-1.
Korrespondenz:

Prof. Dr. med. Edouard Battegay Klinik und Poliklinik

für Innere Medizin

UniversitätsSpital Zürich

Rämistrasse 100

CH-8091 Zürich
Chronische Krankheiten und Multimorbidität sind ein zentrales Thema für Hausärzte und Spitalinternisten. Die persönliche Erfahrung im beruflichen Alltag, dass viele Patienten unter mehreren Krankheiten leiden, wird inzwischen durch viele Studien belegt. Patienten, deren Ersterkrankung erfolgreich behandelt oder in Schach gehalten wird, leben weiter und entwickeln dann vielleicht eine zweite oder dritte Krankheit. Die Patienten werden älter, damit wird sich dieser Trend noch verstärken. Oft wird heute erwartet, dass die Behandlung nach EvidenceBased Guidelines erfolgt. Diese Guidelines basieren auf grossen Studien, die eine möglichst klar definierte Patientengruppe untersuchen. Die eingeschlossenen Patienten sollen möglich keine Faktoren aufweisen, die eine Aussage «verfälschen». Die Studien berücksichtigen somit Einzelerkrankungen und allenfalls häufige Komorbiditäten. Wie sich die Behandlung verändert, wenn zwei oder mehr Krankheiten vorhanden sind, ist noch wenig erforscht. Hausärzte und Spitalinternisten müssen ihre Therapieentscheide so oft aufgrund ihrer Kenntnisse der Einzelkrankheit, aber auch aufgrund ihrer Erfahrung und Intuition treffen. erlangt damit in den stationären Einrichtungen an Bedeutung. Aber auch Ärzte in der Grundversorgung sind gefordert. Patienten sind einerseits multimorbid, andererseits verkürzt sich die Hospitalisationsdauer laufend. Die Behandlung dieser komplexen multimorbiden Patienten verlagert sich damit teilweise in den ambulanten Bereich. Diese Entwicklung muss zu neuen Behandlungskonzepten für den einzelnen Patienten führen und ist auch für die Planung des Gesundheitswesens von grosser Bedeutung. Sie macht eine Stärkung der Allgemeinen Inneren Medizin, der Hausarztmedizin und der Allgemeinen Medizin in stationären und ambulanten Einrichtungen notwendig.

\section{Optimierte Forschung schafft mehr Klarheit und Sicherheit}

Die Forschung wird in Zukunft ebenfalls stärker das Zusammenwirken der einzelnen Krankheiten untersuchen. Hat ein Patient gleichzeitig zwei verschiedene Krankheiten, können sich Guidelines widersprechen: Antikoagulation bei einem thrombotischen Ereignis und gleichzeitig eine Blutung. Was hat Vorrang? Eine Indikation und gleichzeitig eine

\section{Die Therapie der einen Krankheit kann den Verlauf der anderen Krankheit verschlechtern.}

\section{Neuorientierung zwingend}

Die Planung der Ressourcen für Klinik und Forschung wird neu überdacht werden müssen. Die Spitzenmedizin hat in einzelnen Disziplinen grosse Erfolge erzielt. Doch die Behandlung der realen Patienten beinhaltet mehr als die aktuelle Krankheit. Bei medizinischen oder chirurgischen Interventionen multimorbider Patienten ist die Vor- und Nachbetreuung heute oft komplexer. Der Spitalinternist
Kontraindikation für eine Therapie, zum Beispiel Steroide? Gibt es einen Entscheidungslogarithmus? In der klinischen Forschung stehen Fragen zur Interaktion von Krankheiten im Vordergrund, also wie sich Krankheiten gegenseitig beeinflussen. In der ärztlichen Praxis kommt es häufig zu Situationen, in denen man sich bei widersprechenden Guidelines bezüglich verschiedenen Krankheiten zu Entscheidungen durchringen muss, die nicht wirklich befrie- 


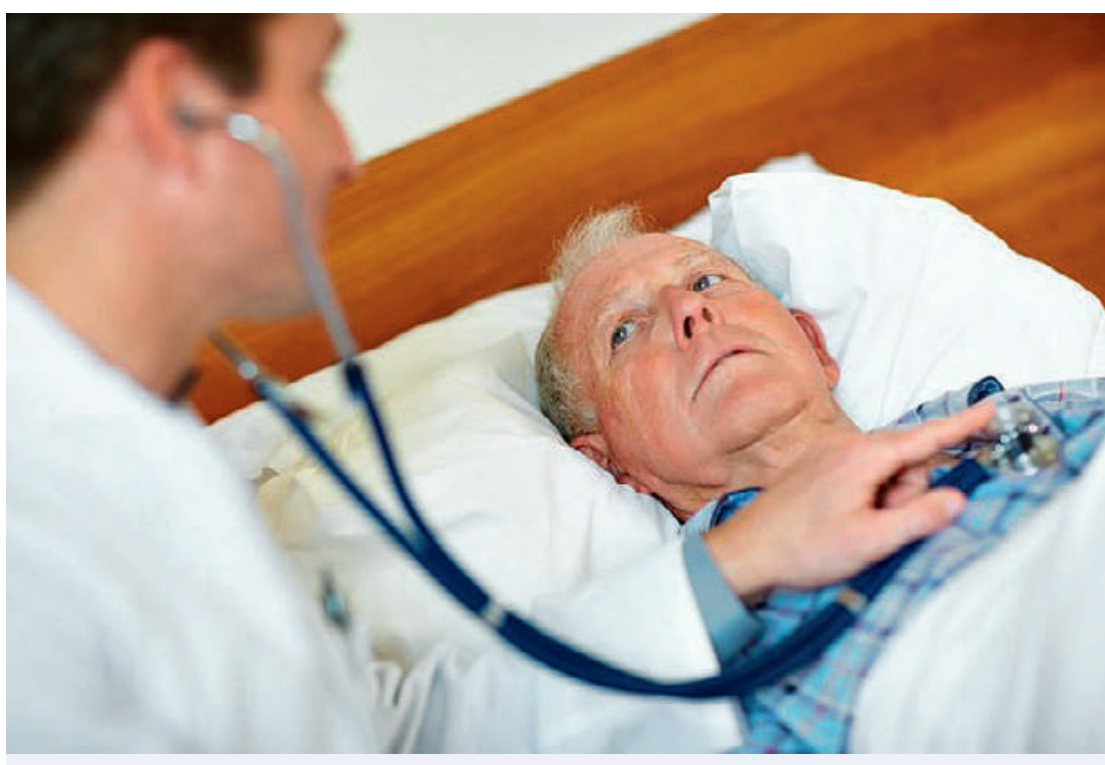

Mit dem Alter steigt die Zahl der Komorbiditäten.

digen. Unter Umständen kann die Therapie der einen Krankheit eine andere Krankheit verschlechtern. Solche Überlegungen sind viel häufiger als Fragen zu Interaktionen verschiedener Medikamente.

\section{Nach stürmischen Erfolgen in der Spezialisierung erfolgt eine Rückbesinnung auf die Zusammenhänge.}

\section{Neue Zielsetzungen fordern innovative Lösungsansätze}

Die Forschung in der Multimorbidität wird in verschiedenen Gebieten vorangetrieben. Die Epidemiologie soll uns ein genaueres Bild des Problems geben, auch wenn sich die absoluten Zahlen aufgrund der sich wandelnden Bevölkerungsstruktur laufend verändern. Die Definition des Problems ist aber auch für die Gesundheitspolitik unverzichtbar, müssen doch die Ressourcen für die medizinische Versorgung rechtzeitig geplant werden. Epidemiologische Ergebnisse zeigen auf, welche Krankheiten in Clustern, also typischerweise gemeinsam vorkommen oder selten gemeinsam auftreten. Dass nach stürmi- schen Erfolgen in der Spezialisierung eine Rückbesinnung auf die Zusammenhänge erfolgt, ist nicht nur in der Medizin aktuell. Ein ähnlicher Trend hat auch die Biologie erfasst. Nach Jahren der fortschreitenden Spezialisierung gewinnen Fragen zur Bedeutung des Zusammenspiels komplexer Systeme an Bedeutung. Dieses Zeichen der Zeit hat die ETH erkannt. Deshalb wurde vor wenigen Jahren ein Lehrstuhl für Systembiologie an der ETH geschaffen, der sich ebendiesen aktuellen und brennenden Fragen stellt.

Unsere Klinik (Klinik und Poliklinik für Innere Medizin, USZ) hat nun einen Schwerpunkt der Forschung auf die Multimorbidität gelegt und das Netzwerk Multimorbidity (www.multimorbidity.net) gegründet. Es ist ein offenes, interdisziplinäres wissenschaftliches Netzwerk von Forschern, PublicHealth-Experten, das neben Forschungseinheiten der Medizinischen und Philosophischen Fakultät der Universität Zürich auch weitere Institutionen umfasst. Dieses Netzwerk hat nun die Anerkennung der Universität Zürich als Kompetenzzentrum Multimorbidität erhalten und ist zudem in einem neuen universitäreren Forschungsschwerpunkt integriert.

Multimorbidität und chronische Krankheiten fordern von Ärzten, insbesondere von Generalisten, komplexe diagnostische und therapeutische Überlegungen, die es zu erforschen gilt. Ein Thema, das uns in Zukunft noch viel beschäftigen wird.

\section{SGIM-Jahresversammlung 2013 - Thema Multimorbidität und neue Programmstruktur}

Chronische Krankheiten und Multimorbidität, ein wachsendes Problem, stehen im Zentrum der SGIM-Jahresversammlung vom 29.-31. Mai 2013 in Basel. Die Jahresversammlung 2013 wird das Thema aus medizinischer Sicht beleuchten, aber auch die Folgen der Multimorbidität für die Planung der medizinischen Versorgung diskutieren. Geändert wird 2013 auch die Programmstruktur. Ziel ist, die Übersicht wesentlich zu verbessern und die unterschiedlichen Bedürfnisse der Teilnehmer zu erfüllen. Zusätzliche Informationen sind zu finden unter www. congrex.ch/sgim2013 oder www.sgim.ch/de/ veranstaltung/sgim-jahresversammlung 2013. 